\title{
Erratum to: Ultraviolet visual sensitivity in three avian lineages: paleognaths, parrots, and passerines
}

\author{
Zachary Aidala • Leon Huynen • Patricia L. R. Brennan • Jacob Musser • \\ Andrew Fidler • Nicola Chong • Gabriel E. Machovsky Capuska • \\ Michael G. Anderson · Amanda Talaba - David Lambert - Mark E. Hauber
}

Published online: 15 September 2012

(C) Springer-Verlag 2012

Erratum to: J Comp Physiol A (2012) 198:495-510

DOI 10.1007/s00359-012-0724-3

In "Materials and methods", under heading "SWS1 sequencing", the two reverse primers mentioned in the last sentence of the first paragraph under sub-heading "Passerines" were published with errors. The correct sentence should read as "We designed two forward primers (SWS1_F1: 5'-CSCC CACGTGGGCCTTCTACC-3'; SWS1_F2: 5'-GTACCAC ATCGCSCCCATGTG- $3^{\prime}$ ) and two reverse primers (SWS1_ R1: 5'-GTGCCCACCGTGTACCAGTC-3'; SWS1_R2: 5'-C ASGTGGCCRCSACGACCAGC-3')".

The online version of the original article can be found under doi:10.1007/s00359-012-0724-3.

Z. Aidala $\cdot$ M. E. Hauber

Biopsychology and Behavioral Neuroscience Program,

The Graduate Center of City University of New York,

365 Fifth Ave, New York, NY 10016-4309, USA

Z. Aidala $(\square) \cdot$ M. E. Hauber

Department of Psychology, Hunter College of City University of New York, 695 Park Avenue, New York, NY 10065, USA e-mail: zaidala@gc.cuny.edu

\section{Huynen · D. Lambert}

Griffith School of Environment and the School

of Biomolecular and Physical Sciences,

Griffith University, Brisbane,

QLD 4111, Australia

\section{P. L. R. Brennan}

Department of Biology, University of Massachusetts,

Amherst, MA 01003, USA

\section{J. Musser}

Department of Ecology and Evolutionary Biology,

Yale University, New Haven, CT 06520, USA

\author{
A. Fidler \\ Cawthron Institute, Nelson, New Zealand \\ N. Chong \\ School of Biological Sciences, University of Auckland, \\ PB 92019 Auckland, New Zealand \\ G. E. Machovsky Capuska \\ Nutritional Ecology and Coastal-Marine Research Groups, \\ Institute of Natural Sciences, Massey University, \\ Auckland, New Zealand \\ M. G. Anderson \\ Behaviour, Ecology and Conservation Group, \\ Institute of Natural Sciences, Massey University at Albany, \\ Auckland, New Zealand \\ A. Talaba \\ Lab of Ornithology, Cornell University, \\ Ithaca, NY 14850, USA
}

